\title{
"We Had to Rely on Each Other": Voices of Latinx Foster Youth with Experiences in Care with Siblings
}

Isabella B. Ginsberg

Portland State University

Follow this and additional works at: https://pdxscholar.library.pdx.edu/mcnair

Part of the Latina/o Studies Commons, Race and Ethnicity Commons, Social Justice Commons, and the Social Work Commons

Let us know how access to this document benefits you.

\section{Recommended Citation}

Ginsberg, Isabella B. (2021) "'We Had to Rely on Each Other": Voices of Latinx Foster Youth with Experiences in Care with Siblings," PSU McNair Scholars Online Journal: Vol. 15: Iss. 1, Article 2. https://doi.org/10.15760/mcnair.2021.15.1.2 
"We Had to Rely on Each Other": Voices of Latinx Foster Youth with Experiences in Care with Siblings

\author{
By \\ Isabella Ginsberg
}

An undergraduate honors thesis submitted in partial fulfillment of the requirements for the degree of

\title{
Bachelor of Arts
}

in

\section{University Honors}

and

\section{Social Work}

\author{
Thesis Advisor \\ Jessica Rodriguez-JenKins, PhD, LICSW
}

Portland State University 


\begin{abstract}
This study used a mixed-methods exploratory design with a demographic questionnaire and interview components that answers the research question: How do various factors affect the experiences of Latinx foster youth in care with siblings? Participants were invited to be interviewed either in person or over the phone. A constant comparative analytic method was used to analyze the interview transcripts to identify common themes. This exploratory study begins to discuss how social work practitioners and foster placements can better support this specific population of children in care. Findings suggest the importance of listening to foster youth and hearing their voices, understanding the role responsibility plays in familial relationships in the Latinx culture, and culturally responsive and individualized knowledge and care from social workers and foster placements.
\end{abstract}




\section{Introduction}

Relationships between members of sibling groups have been found to impact well-being for children who enter foster care (Herrick \& Piccus, 2005). Being placed in stranger foster care is often challenging and can be traumatic with children reporting confusion, worry, and loss of identity and sense of belonging (Herrick \& Piccus, 2005, Unrau et al, 2008). In the instances where the child welfare system separates siblings from one another during their time in foster care, sibling separation can cause more psychological trauma than the loss of a parent depending on the relationship siblings have with one another (McCormick, 2010). While there is some research that explores the experiences of siblings groups in foster care and other research that examines Latinx children in foster care, there is very little information that looks into the unique experiences of Latinx individuals in care with siblings that also accounts for cultural nuance.

Within the limited research there is surrounding siblings in foster care, the focus is often on placement outcomes and how that influences well-being. It is important to explore further into what those sibling relationships are like while in foster care and how that fits into the dynamics of their family system, especially across cultures. Much of the research available about sibling relationships is based on samples of primarily white, middle class, two-parent families (McGuire \& Shanahan 2010). The lack of representation makes it impossible to adequately prepare competent foster homes and caseworkers for working with families from diverse backgrounds and structures.

For instance, within the Latinx culture, there is a high emphasis on familism (Updegraff et al, 2005). There is not a set definition of familism, however research has characterised it as a "multidimensional construct of core values such as family identification, attachment, mutual 
support, family obligation, and familial interconnectedness.” (Mendez-Luck et al, 2016) In relation to children in foster care, this is shown through the priority placed on family and therefore affects the level of care siblings are expected to have for one another. This is important for foster placements to recognize and understand as new children with unique sibling dynamics enter their care. More studies are emerging with insight into sibling dynamics of families coming from ethnic-minority backgrounds but there is continuous room for growth as sibling experiences vary significantly by ethnicity and family structure.

\section{Theory}

As previous research shows, the relationships and support circle in which a foster child has is important. For many foster youth in care with siblings, their sibling is the only constant they have when moving from placement to placement. Things such as their school may change, their primary care physician may change, and their local park might change leaving those sibling bonds especially crucial to keep connected.

The best way to represent their relationships and links to their communities is through ecological systems theory (Bronfenbrenner, 1979). There are five environmental systems present in an individual's life. The first level is the microsystem and this consists of someone's direct environment that they have direct social interactions with. The second is the mesosystem which includes the relationships betweens someone's microsystems. For foster youth, this may present itself as their school interacting with their caseworker to design an independent learning plan. The third level is the exosystem in which structures and people function independently but nonetheless have an impact on the individual and the context of their experiences. An example of this in foster youth experiences is with parents who have frequent interactions with the criminal 
justice system that then indirectly affect the well-being of that foster youth. The fourth level is macosystem which includes the cultural context of someone's experiences. Leaving the fifth level to be the chronosystem which includes major life transitions. For foster youth, this can include the transition into care or the separation from a sibling. This shared understanding is helpful in examining how ecological systems change for a foster youth when they move from placement to placement and the potential impact it has. In relation to Latinx sibling groups in this study, this can help showcase the interplaying levels of microlevel sibling experiences and their meaning in macro contexts that are intricately linked with family context and cultural values across and within societies (Weisner, 1993).

\section{Objectives}

The first objective of this study was to identify the factors affecting the experiences of Latinx youth who have experiences in foster care with siblings. Focus was placed on family dynamics in relationships with siblings before care, during care, and after time in care and expectations placed on them by parents and caregivers. The second objective was to explore the variables associated with improvements for the field of child welfare in relation to working with Latinx youth. Areas surrounding training, shared cultural background of caseworkers and foster placements, and access to individualized case management were emphasised. This study does not intend to show causation or correlation, but attempts to start the conversation for future research to further support this population.

\section{Methodology}

\section{Sample}


The sample came from Oregon and California residents. All five participants had their foster care experiences in Oregon. In order to participate, participants met the following criteria; Latinx, experience in foster care, part of a sibling group in foster care, and age 18+. To do outreach, flyers were posted in public spaces on the Portland State University campus, and posted on various social media platforms. Emails were sent to various departments at Portland State University such as the Department of World Languages and the School of Social Work and they were asked to disperse them to their electronic mailing lists. The sample characteristics in terms of sex, age, placement type can be seen in the following tables. The response options were text entry, so the options displayed below are the ones reported directly by the participants.

\section{Table 1}

Age

\begin{tabular}{|l|c|c|}
\hline & $\mathrm{n}$ & $\%$ \\
\hline $18-23$ & 1 & 20 \\
\hline $24-29$ & 2 & 40 \\
\hline $30-35$ & 1 & 20 \\
\hline Declined to answer & 1 & 20 \\
\hline
\end{tabular}

\section{Table 2}

Gender

\begin{tabular}{|l|c|c|}
\hline & $\mathrm{n}$ & $\%$ \\
\hline Female & 3 & 60 \\
\hline Male & 1 & 20 \\
\hline
\end{tabular}




\begin{tabular}{|l|l|l|}
\hline Declined to answer & 1 & 20 \\
\hline
\end{tabular}

\section{Table 3}

Ethicic/Racial Identity

\begin{tabular}{|l|c|c|}
\hline & $\mathrm{n}$ & $\%$ \\
\hline Mexican & 2 & 40 \\
\hline Hispanic & 2 & 40 \\
\hline Declined to answer & 1 & 20 \\
\hline
\end{tabular}

Table 4

Family Country of Origin

\begin{tabular}{|l|c|c|}
\hline & $\mathrm{n}$ & $\%$ \\
\hline Mexico & 4 & 80 \\
\hline Declined to answer & 1 & 20 \\
\hline
\end{tabular}

\section{Table 5}

Languages Spoken

\begin{tabular}{|l|c|c|}
\hline & $\mathrm{n}$ & $\%$ \\
\hline English & 4 & 80 \\
\hline Spanish & 4 & 80 \\
\hline Declined to answer & 1 & 20 \\
\hline
\end{tabular}

Table 6

\section{Level of Education}




\begin{tabular}{|l|c|c|}
\hline & $\mathrm{n}$ & $\%$ \\
\hline Associates degree & 1 & 20 \\
\hline College/Some College & 2 & 40 \\
\hline University & 1 & 20 \\
\hline Declined to answer & 1 & 20 \\
\hline
\end{tabular}

Table 7

Age Placed Into Foster Care

\begin{tabular}{|l|c|c|}
\hline & $\mathrm{n}$ & $\%$ \\
\hline $5-8$ & 1 & 20 \\
\hline $9-12$ & 1 & 20 \\
\hline $13-16$ & 2 & 40 \\
\hline Declined to answer & 1 & 20 \\
\hline
\end{tabular}

\section{Table 8}

Numbers of Years in Foster Care

\begin{tabular}{|l|l|l|}
\hline & $\mathrm{n}$ & $\%$ \\
\hline $1-2$ & 1 & 20 \\
\hline $3-4$ & 1 & 20 \\
\hline $5-6$ & 2 & 40 \\
\hline Declined to answer & 1 & 20 \\
\hline
\end{tabular}

Table 9

Number of Siblings 


\begin{tabular}{|l|c|c|}
\hline & $\mathrm{n}$ & $\%$ \\
\hline 3 & 2 & 40 \\
\hline 4 & 2 & 40 \\
\hline Declined to answer & 1 & 20 \\
\hline
\end{tabular}

\section{Table 10}

Seperated from Siblings

\begin{tabular}{|l|c|c|}
\hline & $\mathrm{n}$ & $\%$ \\
\hline Yes & 4 & 80 \\
\hline Declined to answer & 1 & 20 \\
\hline
\end{tabular}

\section{Table 11}

Birth Order

\begin{tabular}{|l|c|c|}
\hline & $\mathrm{n}$ & $\%$ \\
\hline Middle & 3 & 60 \\
\hline Declined to answer & 2 & 40 \\
\hline
\end{tabular}

As displayed in the above tables, four of five participants consented to providing demographics. Of those who shared, three identified as female and one identified as male (Table 2). They ranged from ages 19-31 (Table 1). All of the participants identified as part of a sibling group of 3 or more, ranging from sibling groups of four and five (Table 9). In addition, all participants indicated that they were bilingual in both English and Spanish (Table 5) with a family country of origin of Mexico (Table 4). It is also important to highlight that all participants 
shared that they were separated from their siblings at one point in their duration in care (Table 10). Length in care for these participants ranged from one year to over six (Table 8).

\section{Instruments and Procedure}

As seen in the previous section, the questionnaire was an anonymous demographic questionnaire created on Qualtrics and served as the way to gather specific details about the population such as their age, number of years in care, and birth order. The purpose of using this technique was to build an up-to-date reliable database with variables to showcase the background demographics of all of the participants. The questionnaire comprised of eleven items, all of which were open-ended so participants could indicate how they identified in each of the areas. Information on the aspects regarding the participant's foster care experience was gathered as follows: current age, gender, race/ethnicity, country of origin, languages spoken, level of education, age when placed into care, length in foster care, number of siblings, number of siblings that entered care with them, and lastly where the participant stood in the birth order.

Once participants reached out and expressed interest, an interview was scheduled to be conducted in-person in a private confidential space or via telephone. However, due to the timeline of this project and the broad recruitment, all participated remotely to accommodate out of state participants as well as the COVID-19 public health crisis. The purpose of the interviews in addition to the demographic questionnaire was to allow more in depth understanding and gather more detailed information about their experiences in care as part of a sibling set. Participants were given a consent form via email prior to the meeting time and the interviewer reviewed the consent again over the phone prior to the interview. Once verbal consent to participate and be recorded was given over the phone, the interviewer signed on behalf of the 
participant and signed for themselves as the student investigator. At this time, the interviewee was also sent a virtual Amazon gift card code in the form of $\$ 20$ as their compensation. The interview portion lasted approximately one hour. Participants were also given a complete list of mental health resources in the event that they would need to speak to someone after the interview.

\section{Data analysis}

Data analysis was carried out with ATLAS.ti. Transcripts were completed electronically, reviewed for accuracy, and coded for themes.

Data was analyzed using a constant comparative analytic method, where I moved back and forth from analysis to collection until saturation was reached (Creswell \& Poth, 2018). I first got familiar with the data through transcribing my interviews and comparing them with my notes. Data analysis occurred in a multi-stage process using cumulative coding cycles (Creswell \& Poth, 2018). Stage 1 was initial - or open - coding, which involved examining the text and assigning codes to individual words, phrases, or sections using descriptive coding to summarize concepts (Saldaña, 2016) and in-vivo coding using participants' own words describing their experiences (Creswell \& Poth, 2018). Some of those keywords and topics included identity, relationships, and listening. In Stage 2, data were themed, which involved immersion in the data (Saldaña, 2016). Once these codes were all identified, I proceeded with step three of thematic analysis which was developing themes. Using thematic content analysis in Stage 3 created a summary of primary themes (Ayres, Kavanaugh, \& Knafl, 2003). Themes that emerged expanded on the previously found codes such as the sense of responsibility in those relationships and feeling voiceless in instances they were not listened to. Due to my research goals, this 
analysis approach was most beneficial in producing meaning from my data as it relates to the bigger underlying messages behind the experiences of Latinx foster youth with experiences in care with siblings.

\section{Ethics}

The questionnaires did not contain any data that could identify the participants of the study. The social workers referenced in the interviews were not named as well as the name of the foster placements. The questionnaire was anonymized before processing. Data processing was confidential and in keeping with protection and security measures as approved by the Portland State University Institutional Review Board.

\section{Results}

By conducting a thematic analysis on the data collected from the interviews, three themes were discovered. The sense of responsibility was the most prevalent theme that participants shared as part of their experience in care. This included responsibility and obligation in a variety of capacities- from responsibility to their siblings to supporting their parents financially once they were old enough to have a job. The second theme produced was surrounding social workers and foster home efforts. A sub theme of this included cultural competence. The third theme of being voiceless emerged across interviews. Participants shared foster parents not listening when they would try to advocate for the needs of their younger siblings or caseworkers not believing them when they tried to share negatives of what was happening at their placements.

\section{Responsibility}

In the interviews, responsibility was a large encompassing theme brought up.

Responsibility manifested in a multitude of avenues ranging from direct responsibility of siblings 
in new foster homes, responsibilities of their parent's well-being, to their sibling's well-being now as adults. The following quote is from an interview participant who shared her story of being placed in foster care with a younger sister.

"It was never really like a spoken expectation but I think the fact

that I knew that I was older, I just like felt like I had to take care of my sister. Mainly when when my dad knew that they were going to take custody from him for a while the first time, he told me take care of your sister."

In this quote, a parent placed a direct expectation on a child to take care of their sibling but participants expressed that this sense of responsibility was not always outwardly spoken, but a belief placed on them internally or through their upbringing in the Latinx culture. One participant shared she learned how to cook when she was really young and when speaking about friends, "it doesn't seem like they'd ever had that response type of responsibility at 10." Another shared that it was perhaps her identity as a woman and what she learned from her mother that impacted her felt obligation of care for her younger sibling.

"And even in foster care, my mom said take care of your brother because he was only three. So as a woman, I feel like you know, you have a lot of pressure on you even though they don't tell you. It's like an instinct, like, what would my mom do? And my mom's a woman so I reflect that. That's all you know, you know?"

Responsibility can be further broken down into who and what the participants felt responsible for. Primarily, responsibility was focused on caring for siblings, particularly in new placements. Three of the five participants shared a specific story in which they were directly responsible for meeting the needs of a sibling while in a foster home. Examples of specific needs 
provided by these siblings included food, sense of safety, and hygienic duties. Unfortunately, in some foster homes attempting to provide these were not always perceived well by foster parents with one participant sharing an instance where she was reprimanded for "sneaking" into the kitchen to get food for her younger brother. She described the situation as "breaking her heart" because "[her] brother went to sleep crying which broke my heart because I always tucked him in and told him he would be fine."

Another participant shared that losing that sense of control in being able to know for certain if she would be able to provide for her siblings was difficult. Before entering care, she and an older sibling cared for younger siblings by cooking and making sure they were clean.

"I was like the mom of all of us. My sister would make sure that we were all clean and remind us of things like that. And I would always cook, and my brother would just kind of help us out with whatever we asked for. When we entered foster care, all of that obviously went away. I felt like I didn't have control to make sure that they were fed anymore. I didn't have control to make sure that they were safe or tucked in at night. So that was very hard for me." It is in this sense of responsibility, that it is clear the level of connections siblings share with another. Participants shared siblings relying on them for things, even now as adults. One participant shared she did not remember how she even learned how to do independent tasks such as paying bills but had to figure it out due to necessity. Now as adults, her siblings come to her seeking support in those things.

"I still have that role that I have to be the mom for the three of us and think of things ahead. And then anyway, I don't know how I ever ended up figuring out 
how to pay bills, how to rent an apartment, how to apply for things. But I ended up figuring it out. And they would always seek me for all of that, like, how do I apply for my license? How do I do this? How do I pay back? You know, where should I apply for school? Where do I go for work?"

Even to this day, participants shared feeling a great sense of pressure to make sure they are doing okay.

Beyond responsibility for siblings, participants shared instances in which they played a role in their parent's responsibilities. A few shared instances in which they were expected to work and provide financial support once they were old enough, with two participants even sharing instances of working before then because their parents would bring them to their work. Even as a child, one participant shared feeling responsible for checking-in on her father's well-being during their DHS visits.

\section{Case worker and Foster Home Efforts}

In addition to responsibility, the topic of efforts made by caseworkers and foster parents affected these individuals time in care. Things from efforts made to maintain sibling connection in instances where siblings were separated to efforts to provide proper hygiene supplies were discussed. Furthermore, the importance of cultural competence in both the workforce handling their cases and their everyday living situations in their foster homes was an underlying pattern in the analysis.

All participants shared at least one personal encounter they had with a caseworker that affected their experience. For most, the experiences that stood out were the positive ones. Positive experiences were characterized by feeling understood in terms of their cultural 
upbringing and feeling a sense of genuine care from their caseworker. One participant shared that "my caseworker was Mexican so that I liked. I felt like she could understand me" speaking to how the direct connection of her caseworker sharing her Mexican identity affected her time in care.

In addition, participants expressed higher need for cultural competence in their foster placements with specific reference to meal and food choices. The way food is viewed and eaten varies from culture to culture, and for one particular participant this affected their foster home experience. She shared on multiple occasions she was given food by a foster parent and did not understand what it was or how to eat it. She had to learn and then teach her younger sibling that it was okay to eat.

“The people that were our foster parents just didn't know our languages or you know our culture because one time they gave us fruit roll up and I had no idea what it was. They had like ripped it up into pieces and they're like, 'you guys have it good, here's two pieces of fruit roll ups' and I didn't know what to do with it because I was like my mom never gave it to us. They had another child who ate it so I told my brother 'puedes comer'."

In another instance, this participant felt made fun of by her foster parents because they gave her and her younger sibling toast with beans and said "this is close to a tortilla, you'll be fine". For many, food is a comfort and when foster parents can provide familiar cultural foods it can make a difference. For many foster youth, all things familiar and comfortable are taken away so by keeping these comfort foods change can be more gradual. 
Also, religion can be classified as part of someones culture and this was brought up in a few interviews. Having foster parents that understood their religious beliefs and preferences provided a sense of comfort. For a particular individual, this was also a positive experience with a caseworker because they listened when they requested a placement that practiced the same religion.

\section{Feeling Voiceless}

Language such as "what they thought was best for us" was used continuously across the interviews to describe ways their case was handled that did not align with their own needs and wants. Participants shared being placed in foster homes who thought adoption for a few of the siblings was best, but not for the others. One particular participant shared this was difficult for her because "she could not be with them to protect them." For many of these individuals, their siblings were the only constant in their life and not respecting their desire to stay together was difficult.

In the unfortunate reality for many foster youth, the ignorance from foster parents and caseworkers can be traumatic and damaging. In describing an instance in which she was locked in a room for sneaking out of her room to get food for her younger sibling a participant said, "nobody answered me [when I said I had to go to the bathroom], they thought I did something evil and so they didn't even let me explain myself'. As a result, she was forced to relieve herself in the room. In another instance, a handmade gift from a birth parent was sold by her foster parents without discussing it with her. When she was given the opportunity to tell her caseworker what happened, the caseworker did not believe her.

\section{Discussion}


A goal of this research was to emphasize areas of previous research that have been overlooked or not fully considered such as the Latinx experience in the foster care system. As previously shared, the model of primarily white, middle class, two-parent families is often found in foster care research (McGuire \& Shanahan, 2010). This study placed a new focus on aspects such as family dynamics in relationships with Latinx siblings before care, during care, and after time in care as well as expectations placed on them by parents and caregivers. The basis of the cultural context of these unique experiences agrees with the literature that understanding unique experiences on the macro and micro level cannot be based on a few samples of a certain family type (McGuire \& Shanahan, 2010).

In these stories, it is evident that the foster youth have ever changing ecological systems that affect their experiences in care. Their immediate microsystem comprises their siblings, caseworkers, foster parents, and biological parents. In the cases of these participants, the actions made by their biological parents (exosystem) is affecting the relationship these siblings have by tightening their bond. On the macro level, societal expectations in the realm of gender and culture affect to which degree they feel a sense of responsibility. Similarly, their cultural and religious contexts in the macrosystems did not always match up with their needs. Placements do not always understand shifts in those systems and that has the potential to further take away that sense of familiarity for these youth and leave them feeling a loss of control.

The results of this study show that these Latinx foster youth were not given enough agency and voice in their experiences. They held a significant amount of pressure and responsibility during their time in care and that continued into their lives as adults. Despite this, they were not fully believed when they tried to advocate for themselves or their siblings. 
Participants shared that they realized they could not always have their ideal situation, but it helped when caseworkers explained why things were the way they were or made it clear the efforts they were making. Actions like these did not render them voiceless but gave them a sense of agency in their life because there was more communication about things directly affecting their life. In the child welfare system, the best interests of foster youth are decided by professionals based on laws and regulations with little effort to involve youth in those decisions making simple things like this to give them voice all the more important (Cristadoro, 2009).

As much as it is possible, caseworkers able to be constant throughout a foster child's entire time in the foster care system was expressed as being important. Even years later, a participant expressed having the same caseworker throughout their entire time as "the best thing ever". The participants shared that this allowed the caseworker to see everything that was happening and help them through it.

One thing that came out of this study that was not planned for, was the impact being a foster child has on their parenting style. Of the five participants, three shared how being a mother has been affected by the way they were brought up in foster care.

"The way that I raise my son is not the way that I was raised. So I think that perhaps having that experience getting [my dad's] custody taken away, helped me understand that. And of course, it's not something I want my son to go through and do I want to go through but makes me conscious about how I raised my son." This just goes to show the lasting impact foster care experiences have on an individual.

\section{Limitations}


While there are many strengths of this study, a limitation was the difficulty recruiting participants. Because the sensitive nature of this interview and specified criteria, it is believed to have affected recruitment efforts. If this study were to be replicated, the ideal would be to have more participants to perhaps get a larger understanding of the unique experiences of Latinx foster youth.

\section{Policy Implications and Future Research}

This study is not able to show causation or correlation, but based on the findings there are a few recommendations that should be considered further. Going forward, a recommendation would include measures in place to ensure quality caseworkers. For Latinx foster youth, quality would look like hiring bilingual and bicultural caseworkers able to speak the language of their families and be familiar with the cultural customs of the Latinx community.

Another way to support Latinx individuals in care would be to offer individualized case management. This would mean that each person in the family unit is respected as an individual person with their own unique needs. Individualized care is needed that reflects who the individual is and meets their particular needs. This requires background knowledge of the foster youth so caseworks should be prepared to talk one on one with members of the family and take action depending on what those individuals express as needs. In practice, this may include ensuring every member of the sibling group has their own set of toiletries or offering specific resources as they pertain to each sibling.

When asked about what they wish professionals in the field understood about their experiences as a Latinx individual in care, the answers varied. It is important to highlight every single response and story. We cannot go back in time and change the circumstances and 
experiences for these foster youth, but we can make sure they are heard and their stories are making an impact on the future of child welfare work. Their responses are provided below:

The first participant would like people to know:

"I think that what means the most to a child at that time, is that they take the time to make that child feel like they matter and they're important. Sit with them and just have a conversation like, 'Hey, you know whether or not you feel like you have control over this, I'm here and I care for you. You're not here just because you don't have anywhere to live.' So just to have someone to dedicate their time for these children I think is going to matter the most and make a big difference because a lot of us are neglected and whatever experiences might have caused kids to be taken away from home to be in a better safe place, I still think that love will always make things better and that touch of affection of like having someone hug you or even sleeping next to you, anything that makes you feel okay."

The second participant would like to share:

"So with any foster kid that goes through that, you know, it's really important to give them a place where they're included, they already feel like they're different."

The third participant would like people to know:

"They need to investigate these foster parents a lot better, I had a lot of horrible foster parents."

The fourth participant would like people to know:

"More home visits could be done. If anything, I think that would help kids that are still in foster care. If their caseworkers would visit them more often."

The fifth participant would like people to know: 
"I wish they would have cared more about the loss of connection I had with my identity as a Latinx individual. My mother was not born in the United States, so she was all I really had to help me connect with my roots back in my family's home country. I felt like I was being stripped of my family's traditions, my family's food recipes, my culture, my ability to say "this is my grandma's recipe" and so much more. I hope they can somehow change that in the future and help Latinx foster youth and other youth of color maintain a sense of community with people who share the same culture/identity as them."

\section{Conclusion}

The objectives of this study sought to identify the factors affecting the experiences of Latinx youth who have experiences in foster care with siblings and explore areas of improvement for the field of child welfare. This study brings attention to the continued work that needs to be done to better support the needs of Latinx individuals with experiences in foster care with siblings and their families. The child welfare system is historically a reactive system designed to remove children from their homes when things are deemed not safe for them, rather than provide preventative supports to families before that has to happen.

This research should encourage professionals and stakeholders in child welfare to recognize the systemic issues in place that prevent Latinx families from flourishing as a family unit and find ways to disrupt this so they can make unbiased decisions when removing a child from their biological home and placing them into foster care. If after all options have been exhausted and a child needs to enter care, substantial efforts need to be made in maintaining sibling relationships and cultural connection. This study is a reminder that foster youth need to be in control of their stories and have their voices heard. 
Ginsberg 23

\section{Funding}

Funding for this project was provided by the McNair Scholars Program at Portland State University and through the Wessinger Foundation Scholarship provided by the University Honors College at Portland State University. 
Ginsberg 24 


\section{References}

Ayres, L., Kavanaugh, K., \& Knafl, K. A. (2003). Within-case and across-case approaches to qualitative data analysis. Qualitative Health Research, 13(6), 871-883. doi:10.1177/1049732303013006008

Bronfenbrenner, U., 1979. The Ecology Of Human Development. Cambridge, Mass.: Harvard University Press.

Creswell, J. W., \& Poth, C. N. (2018). Qualitative inquiry and research design: Choosing among five approaches (4th ed.): Sage Publications.

Cristadoro, Nancy A., "Children of Foster Care: Elevating their Voices" (2009). All Regis University Theses. 659. https://epublications.regis.edu/theses/659

Herrick, M. A., \& Piccus, W. (2005). Sibling connections: The importance of nurturing sibling bonds in the foster care system. Children and Youth Services Review, 27(7), 845-861. doi:10.1016/j.childyouth.2004.12.013

Mendez-luck, Carolyn \& Applewhite, Steven \& Lara, Vicente \& Toyokawa, Noriko. (2016). The Concept of Familism in the Lived Experiences of Mexican-Origin Caregivers. Journal of Marriage and Family. 78. n/a-n/a. 10.1111/jomf.12300.

McCormick, A (2010) Siblings in Foster Care: An Overview of Research, Policy, and Practice, Journal of Public Child Welfare, 4:2, 198-218.

Mcguire, S., \& Shanahan, L. (2010). Sibling Experiences in Diverse Family Contexts. Child Development Perspectives, 4(2), 72-79. doi:10.1111/j.1750-8606.2010.00121.x

Saldaña, J. (2016). The coding manual for qualitative researchers (3rd ed.): Sage Publications Unrau, Y. A., Seita, J. R., \& Putney, K. S. (2008). Former foster youth remember multiple 
placement moves: A journey of loss and hope. Children and Youth Services Review, 30(11), 1256-1266. doi:10.1016/j.childyouth.2008.03.010

Updegraff, K. A., Mchale, S. M., Whiteman, S. D., Thayer, S. M., \& Delgado, M. Y. (2005). Adolescent sibling relationships in Mexican American families: Exploring the role of familism. Journal of Family Psychology, 19(4), 512-522. doi:10.1037/0893-3200.19.4.512

Weisner, T. S. (1993). Ethnographic and ecocultural perspectives on sibling relationships. In Z. Stoneman \& P. W. Berman (Eds.), The effects of mental retardation, disability, and illness on sibling relationships: Research issues and challenges (p. 51-83). Paul H. Brookes Publishing. 